\title{
Local Variations in the Surface Plasmon at Al Grain Boundaries and its Effect on the Optical Properties of Al Nanostructures.
}

\author{
Andrew Thron ${ }^{1}$, C. G. Bischak ${ }^{2}$, Scott Dhuey ${ }^{1}$ and Shaul Aloni ${ }^{1}$ \\ ${ }^{1}$ Molecular Foundry, Lawrence Berkeley National Laboratory, Berkeley, CA 94720 \\ ${ }^{2}$ Deparment of Chemistry, University of California, Berkeley, Ca 94720
}

Localized Surface Plasmon Resonances (LSPRs) are collective oscillations of free charge carriers which are driven by an external electric field. Light can be confined to nanoparticles and lithographically defined structures smaller than it's wavelength by coupling the electric field to LSPRs. The dispersion behavior of the LSPR is bound by the asymptotic limit, the surface plasmon frequency:

$$
\omega_{s p}=\frac{\omega_{p}}{\sqrt{1+\varepsilon_{2}}},
$$

where $\omega_{\mathrm{sp}}$ is the surface plasmon frequency, $\omega_{\mathrm{p}}$ is the bulk plasmon frequency, and $\varepsilon_{2}$ is the dielectric function of the layer above the metal surface. With an inert metal such as $\mathrm{Au}$, the surface plasmon frequency will stay constant in vacuum. Therefore, inherent variations to the LSPRs, in Au nanostructures, are largely associated with changes in shape, microstructure, and surface roughness. However, Knight et. al. observed a different behavior in Al disks, where the dipolar LSPR energy varied depending on the thickness of Al oxide layer [1]. Shifts in the LSPR were attributed to changes in the dielectric function of the oxide layer, which caused the surface plasmon to shift in energy. Decoupling the roll of shape, microstructure, and composition is necessary for successful design of Al plasmonic devices. Monochromated EELS in STEM has shown to be an effective technique in studying how microstructural changes influence the behavior of LSPR due to the high spatial resolution [2]. In this study Monochromated EELS and STEM shows that the surface plasmon varies locally across lithographically defined Al triangles, especially at the triple point between the grain boundary and the surface oxide layer.

Lithographically defined Al triangles were created on $30 \mathrm{~nm}$ thick $\mathrm{Si}_{3} \mathrm{~N}_{4}$ membranes (Figure 1a). Using EELS, a decrease in the bulk plasmon peak energy was observed to occur at the grain boundaries (Figure 1b). This is attributed to a decrease in the charge carrier density at the grain boundaries due to a decrease in atomic density. A concurrent increase in the surface plasmon peak energy is also observed at the grain boundaries (Figure 1c). Using the relationship in equation 1 , together with the measured surface and bulk plasmon peak energies, the dielectric function $\left(\varepsilon_{2}\right)$ is observed to vary across the Al triangle (Figure 1d). Specifically, $\varepsilon_{2}$ is observed to decrease at the triple points, where the grain boundaries meet the oxide layer. Changes to the LSPR supported by the Al triangle were not observed to due to their delocalized behavior (Figures 1e-i). However, local variations to the surface plasmon should contribute to scattering of the LSPRs.

This study attempts to correlate the chemical and structural affects to the behavior of LSPRs. Further characterization of the oxide layer is needed in order to understand how variations in the oxide layer over the triple point and over the grain interior change $\varepsilon_{2}$. Moreover, in-Situ and ex- 
situ annealing experiments will be used to study the effect grain boundary density and structure have on the surface plasmon, and ultimately correlating how the surface plasmon contributes to LSRP scattering.

\section{References:}

[1] Knight M.W. et. al., ACS Nano, 8 (2014), 834-840

[2] Bosman M. et. al., Sci. Rep., 4 (2014), 5537
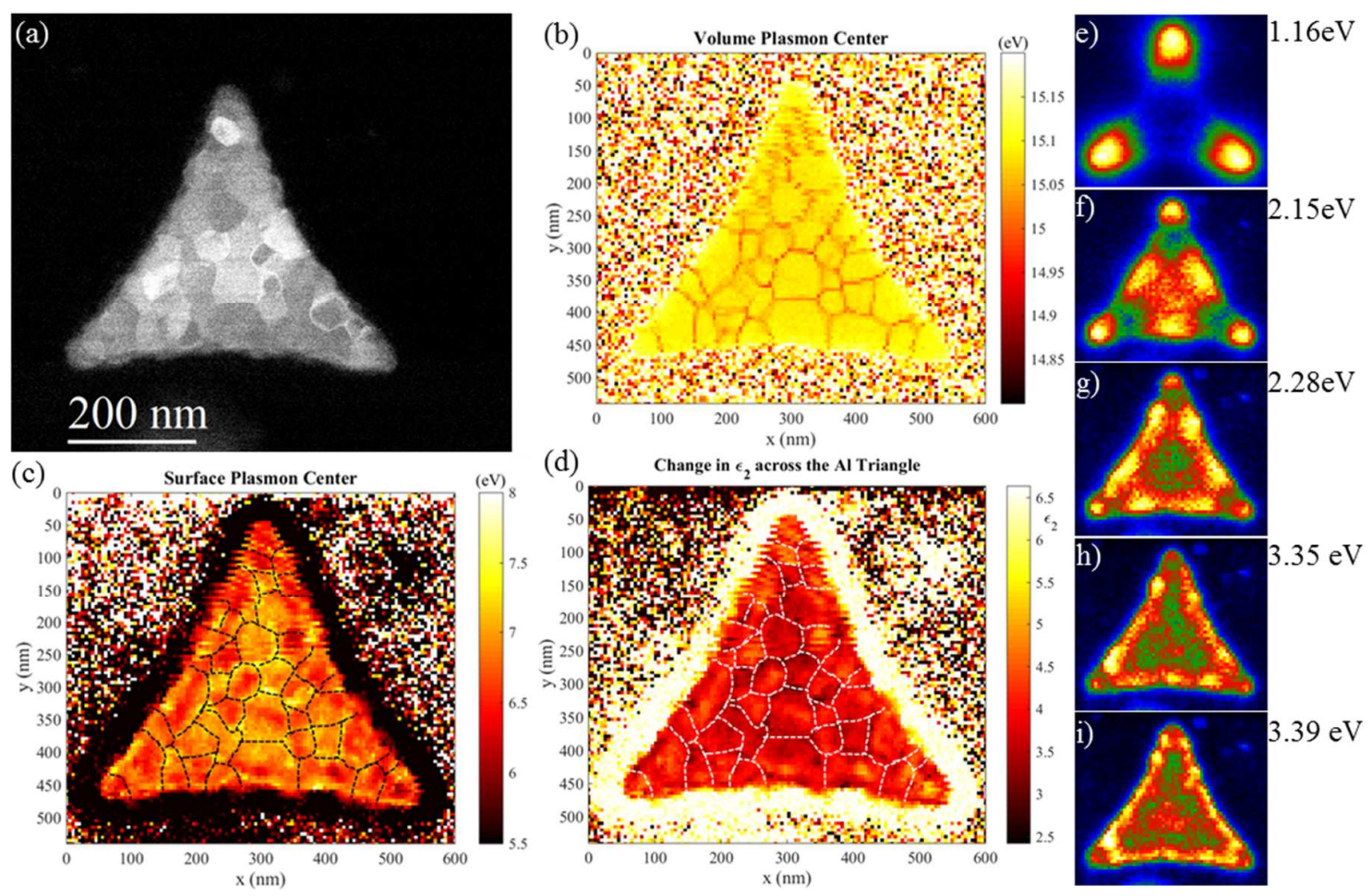

Figure 1. (a) Annular Dark Field image of lithographically defined Al Triangle. (b) Center of the Gaussian function fitted to the volume plasmon peak (c) Center of the Lorentzian function fitted to the surface plasmon peak. The overlaid black outlines are the positions of the grain boundaries observed in (b). (d) The change in the dielectric function $\varepsilon_{2}$, calculated from equation (1). The white outline highlight the positions of the grain boundaries. (e-i) Local Surface Plasmon resonant modes supported by the $\mathrm{Al}$ triangle. 